\title{
Breast Cancer in Senior Patients: Role of Comprehensive Geriatrics Assessment and Comorbidities
}

\author{
Marco Ruiz*, Charles Cefalu and Tom Reske
}

Department of Medicine, Section of Infectious Disease, Louisiana State University, USA

\begin{abstract}
Background: Breast cancer is the most common cancer in women in the United States and Europe. Comprehensive geriatrics assessment (CGA) is a key tool for geriatricians and accomplishes appropriate stratification of senior patients into independent and frail patients. CGA involves a thorough assessment of different domains including psychical and psychological spheres (functional assessment, cognitive, depression, nutrition status, social functioning). Some reviews have explored the use of CGA in elderly breast cancer patients but more studies comparing the CGA and abbreviated versions in elderly breast cancer patients are needed.
\end{abstract}

Methods: We searched PubMed, restricted to the past 10 years, with the terms "breast cancer", "elderly patients", "older patients and breast cancer", "Comprehensive geriatrics assessment","frail breast cancer patients", "comorbidities and breast cancer," and "geriatric syndromes".

Discussion: CGA may detect multiple problems in senior cancer patients. Moreover, CGA is an excellent too to stratify patients into healthy independent and frail patients. It gives an insight on first line treatment and adjuvant chemotherapy options in this population. Despite its benefits issues such as lack of time, length of the test, difficulty to interpret results and need for regular follow up, prevent its use in busy oncology practices. Shorter versions of the CGA have been tried but these tools face problems with generalizability and validity. Comorbidities, frailty status, and the presence of geriatric syndromes have been directly related to increased morbidity and mortality in elderly breast cancer patients.

Conclusion: Breast cancer prevalence continues to increase specially in seniorpatients. Comprehensive geriatrics assessment has been advocated as a screening and follow up tool for senior patients. In senior breast cancer patients, CGA may help stratify patients who may develop adverse reactions to treatment strategies. The roles of comorbidities, geriatric syndromes, and frailty status and their impact on survival need to be elucidated.

\section{Introduction}

Breast cancer is the most common cancer in women in the United States and Europe. Aging is a very important risk factor for developing breast cancer. With aging of population it is expected that the prevalence of breast cancer will increase in the incoming years in the senior population ( $>65$ years). In fact, it is expected that by the year $2035,60 \%$ of new patients are 70 years or older [1]. Therefore a thorough evaluation of elderly breast cancer patients is be needed to properly stratify this population into those who may respond adequately to treatment strategies and others who may present with complications associated with treatment [1-5].

Comprehensive geriatrics assessment (CGA) is a key tool for geriatricians [1-4]. It involves a thorough assessment of different domains including psychical and psychological spheres (functional assessment, cognitive, depression, nutrition status, social functioning [6]. It has been used with success in geriatric practice for evaluation of senior patients by stratifying patients based in functional status (independent, partially dependent, and fully dependent). A recent meta-analysis study has showed that when CGA is used mortality of acutely hospitalized elderly patients decreases at 1 year after discharge [6].

The applicability of comprehensive geriatrics assessment in other medical specialties has been suggested with success in certain occasions but with some obstacles in other cases [3,4,7-9]. In oncology practice the value of comprehensive geriatrics assessment has been reviewed by many authors who do agree of the need to incorporate the comprehensive geriatrics assessment into oncology practice [1-3,5,79].
Many obstacles such as lack of time, lengthy test, difficulty to interpret results and need for follow regular follow up; have been identified when trying to incorporate CGA in clinical oncology practice $[1-3,7,10,11]$. Some attempts to shorten the CGA were reviewed and published in the literature. These shorter versions face issues related to generalizability, reliability, and validity $[3,4,10,11]$.

Even though the use of CGA is highly recommended for senior cancer patients, it is not widely used in oncology practice. Assessment of senior patients in general is performed with other oncology-based tools such as the ECOG and the Karfnosky index and performance status [7]. These tools partially determine functional ability of senior patients but important aspects of geriatrics assessment such as cognitive function, social functioning, nutritional status, presence of depression, and medication interactions are missed $[4,6,12]$.

*Corresponding author: Marco Ruiz, MD, MPH, Department of Medicine, Section of Infectious Disease, 136 South Roman St., 2nd floor, New Orleans, LA 70112 USA, Tel: 504-903-2926; Fax: 504-903-5313; E-mail: mruiz@Isuhsc.edu

Received August 02, 2011; Accepted October 25, 2011; Published October 27 2011

Citation: Ruiz M, Cefalu C, Reske T (2011) Breast Cancer in Senior Patients: Role of Comprehensive Geriatrics Assessment and Comorbidities. J Cancer Sci Ther S2. doi:10.4172/1948-5956.S2-002

Copyright: () 2011 Ruiz M, et al. This is an open-access article distributed unde the terms of the Creative Commons Attribution License, which permits unrestricted use, distribution, and reproduction in any medium, provided the original author and source are credited. 
Appropriate stratification of senior patients into independent, partially dependent, and fully dependent or frail groups is crucial for determining correct medical interventions [5]. It has been shown that highly functional patients usually respond well to chemotherapy/ radiation/surgery interventions while on the other hand frail patients present with multiple complications as a result $[3,4]$

This paper reviews the role of comprehensive geriatrics assessment in the initial evaluation and subsequent follow up of elderly breast cancer patients, the role of comorbidities and geriatric syndromes in elderly breast cancer outcomes and survival.

\section{Breast cancer management in senior patients}

Current recommendations advocate an individualized screening in senior patients based on functional status and estimated life expectancy $[1,2,13,14]$. The American Geriatrics Society recommends annual screening mammography up to the age of 85 , if their life expectancy is at least 4 years.

The role of diagnostic mammograms have been discussed in the literature [1,2]. Studies have suggested that senior breast cancer patients should be treated based on fuctional status, comorbidities and prognostic factors and not just based on chronological age $[2,3]$. In addition to the standard of care, mammogram, and ultrasound, MRI screening of contralateral breast in newly diagnosed breast cancer patients showed benefits even in senior patients [10].

Surgery is the mainstay of treatment for senior patients. It has been associated with lower morbidity and longer survival in octogenarians [11]. Interestingly, recent data suggest that women $>70$ were more likely to receive no surgical treatment and undergo mastectomy in some cases $[15,16]$. There is some controversy about the utility of lymph node dissection in senior patients $[16,17]$.

Adjuvant therapy has also been advocated for senior patients. An adjuvant US trial published in 2005 showed that women age 65 or older had an increased overall survival and relapse free survival using standard of care combination chemotherapy versus the single agent capecitabine. This trial confirmed an improved outcome with combination chemotherapy compared to a single agent regimen in the adjuvant setting as it is seen in younger breast cancer patients [18]. Sautler et al. [19] suggest that based on risk stratification, independent senior patients should be given chemotherapy, and radiotherapy as standard adjuvant chemotherapy. Transtuzumab is thereby recommended as adjunct to standard chemotherapy in HER 2 positive disease. Retrospective studies from France confirmed efficacy and safety of standard docetaxel and cyclophosphamide in 110 seniors $(>70$ years) [20]. Kimmick [21] and Garg et al. [22] found similar response and tolerability to standard chemotherapy regimens.

Adjuvant hormonal therapy has been used successfully in adjuvant setting in hormone receptor positive disease. Besidest tamoxifen, aromatase inhibitors have been proven to be well tolerated. The inhibitors are frequently used in elderly patients, although long term data on use in the elderly is not as extensive compared to tamoxifen [23]. It remains important to screen for bone health prior to start of an aromatase inhibitor, as bone density can significantly decline during therapy. Start of bisphosphonate therapy with calcium and vitamin D is recommended if a decline in bone density is diagnosed.

Radiation therapy in the adjuvant setting is also commonly used to prevent local recurrence. Radiation is usually well tolerated. A study by Hughes et al. [24] in patients $>70$ years in early stage disease questioned though the benefit from radiation therapy in this subset of patients. Radiation was shown to prevent local recurrence compared to observation 1 versus $4 \%$, there was no benefit in overall survival.

Targeted therapies for certain breast tumors (HER 2 positive) have been reviewed in the literature. These therapies may significantly allow longer disease-free survival times. These drugs are not free of side effects and in fact may cause significant issues in elderly patients (cardiotoxicity, deep vein thrombosis, gastrointestional and dermatologic). New trials including senior patients are urgently needed to clarify the potential benefits of this group of agents [25].

In summary, clinical trials have not included a good number of elderly breast cancer patients $(>70)$ [3,4] Screening for elderly ( $>70$ years) patients is recommended based on individual patient characteristics. Even though surgery is the mainstay of treatment it appears that surgical options are not offered as frequently as other treatment modalities in this population. Adjuvant therapy and aromatase inhibitors appear to be safe in the independent/functional subset of elderly patients. Targeted therapies need to be further investigated in elderly breast cancer patients.

\section{Comprehensive geriatrics assessment as a tool for a thorough stratification of senior breast cancer patients}

The role and benefits of comprehensive geriatrics assessment has been discussed widely in the literature [8,26-29]. CGA may detect multiple problems in elderly cancer patients [27]. Moreover, CGA is an excellent tool to stratify patients into healthy independent and frail patients [9]. CGA has also been used as a very precise prognostic tool in oncology settings $[30,31]$. In fact this tool helps to adapt cancer treatment for these patients [32].

In busy oncology settings the majority of oncologists use the ECOG (Eastern Cooperative Oncology Group) evaluation tool to stratify (functional and non-functional) patients. Multiple authors have compared CGA and ECOG (Eastern cooperative Oncology Group) evaluation tools and have shown that CGA is a more thorough tool able to detect issues not detected by ECOG $[3,4]$. Therefore, the CGA seems to be the best tool available for evaluation of elderly cancer patients. Unfortunately issues such as the length of the CGA, busy oncology practices, lack of trained staff prevents its use in oncology practice. As a result of this shorter version of the CGA has been suggested.

The role of shorter versions of the CGA has been reviewed in the literature. Aparicio et al. [32], piloted a study of 21 patients over the age of 70 using a mini-geriatrics assessment tool (shorter version of CGA). The aim of the study was to evaluate feasibility of a mini geriatrics assessment to adapt the anticancer therapy. These authors concluded that the mini CGA lead an adaptation of the non-oncology treatment in $72 \%$ of cases and of the social care in $38 \%$ but the oncology strategy was never modified [32]. In a retrospective analysis, Overcash et al. [33] reviewed over 500 charts in patients over 70 year with the intent to validate an abbreviated version of CGA( 15 items ). The study showed a direct correlation between the abbreviated form and the entire CGA. The researchers recommended the abbreviated form as a screening tool for those seniors who would benefit from the entire CGA.

Another shorter version a brief comprehensive self-administered questionnaire (functional status, comorbidity, psychological status, nutritional status, and social support) was conducted in 250 patients in an academic oncology practice. The median time to complete the questionnaire was 15 minutes. Information collected helped to identify 
physical and psychological impairments and other comorbidities [34]. In another study Hurria et al. [26] tested a self- administered brief questionnaire in senior cancer patients (lung, breast, colorectal, and lymphoma) who were receiving chemotherapy in an academic oncology center. The intent of the study was to determine if senior cancer patients were able to complete a self-administered questionnaire, length of time to complete it and the satisfaction with the measure. The questionnaire was an abbreviated form of CGA. Selected areas included functional status, comorbidities, cognition, psychologic, social functioning, social support, and nutrition. A total of 43 patients completed the questionnaire. The mean time to completion was 27 minutes. The majority of patients (78\%) completed it without any assistance. The authors concluded that this abbreviated form might be useful in busy oncology practices but more prospective studies were needed for validity, generalizability, and realiability. The results of these shorter versions encouraged researchers to pursue the development of cancerspecific geriatrics assessments.

Molina-Garrido et al. [35] piloted a brief but not self-administered cancer-specific CGA and determined its feasibility measured by length of time to complete and patient satisfaction. Ninety nine patients completed this instrument. The total amount of time was 12.9 minutes. The majority of patients were satisfied with its length (63.6\%) and the easiness to complete it (69.7\%). The authors recommended further studies for validation of this tool.

CGA has also been used as a tool for screening, diagnosis and follow up purposes in oncology practice. One of the main obstacles is its length and its dissemination in busy oncology practices may be difficult to achieve. Shorter versions have been tried composed of more subjective tools with problems in generalizability and validity $[3,4,26]$.

Some reviews have explored the use of CGA in elderly breast cancer patients. Taira et al. [5] in a review o article, investigated the use of CGA in elderly Japanese breast cancer patients. The authors found that the CGA could be used as a screening and follow up tool but its use has been limited and as a result of this CGA-related outcomes has not been studied properly in clinical trials. The authors recommended conducting clinical studies where the CGA is used in order to determine its applicability and use in oncology practice. Wedding et al in a review article of elderly breast cancer patients found that the CGA gives an insight on the screening, first line treatment, and adjuvant chemotherapy options in this population. The authors recommended the development of more clinical trials where the CGA is used in the evaluation of elderly breast cancer patients.

Owusu et al. [36] in a review of breast cancer in the elderly suggest the important role of the CGA in the care of patients. The authors refer the need for complete evaluation of elderly breast cancer patients before treatment interventions.

Barthelemy et al. [37] in a review of 192 elderly patients with early breast found the impact of the CGA on treatment decision-making in patients over the age of 70 . The authors found that the CGA was not fully integrated in the decision-making process and recommended that this tool should be better integrated in this process. In another review article Albrand et al. [38,39] found the benefits of the CGA in elderly breast cancer patients with early breast cancer. The authors recommended an initial CGA followed by a comprehensive oncological assessment. This dual assessment would define patients who are frail and as a result of this not able to undergo oncological treatments and the ones who are fit enough to undertake oncological interventions.
In summary, the CGA has a pivotal role in the evaluation of elderly breast cancer patients. The lack of clinical trials where the CGA is included has prevented its dissemination into oncology practice. More studies comparing the CGA and abbreviated versions in elderly breast cancer patients are needed.

\section{Role of comorbidities and geriatric syndromes}

The interrelation among comorbidities, geriatrics syndromes, aging, frailty, chronic inflammation and cancer has been reviewed in some reports. Siegelmann-Danieli et al. [40] revised these associations among 992 elderly breast cancer patients. Interestingly, these authors found that the presence of comorbidities may adversely affect breast cancer survival. This association seems to be stage dependent major negative impact of comorbidities on early breast cancer stages. These authors suggested a stage shift with patients with more comorbidities having similar outcomes compared to patients with advanced breast cancer stages

The association of geriatric syndromes and cancer was reviewed by Mohile et al. [41]. In a study of Medicare beneficiaries there was an association between the diagnosis of geriatric syndromes and cancer [41]. Patient with 1 or more geriatric syndromes had worse outcomes in terms of adverse reactions and mortality than patients without geriatric syndromes. Other authors explained these findings by looking into the association of geriatric syndromes and frailty status $[7,42]$.

Comorbidities have been directly related to increased morbidity and mortality in senior cancer patients. In a study of 64,034 patients with a median age of 75 years, a total of 13 comorbidites were found to be related to increased morbidity and mortality. The presence of any of these comorbidities negatively affected survival independent of breast cancer stage and type of tumor [43]. In another study of 159 senior breast cancer patients, more than $65 \%$ had comorbidities the most common being cardiovascular conditions (coronary artery disease and hypertension, and transient ischemic attack) [1].

In another study Girones et al. [44] showed that senior breast cancer patients suffer from more comorbidities on follow up after initial early breast cancer diagnosis. Ninety one patients with an average age of 76 years at time of surgery for breast cancer were identified. The median follow up was 5 years. The study showed that the subgroup of functional patients continued with follow up more frequently than frail patients. Comorbidity was independent of functionality and age [44].

There seems to be a direct correlation among comorbidities, geriatric syndromes and outcomes in senior breast cancer patients. Some authors suggest the possibility of a stage shift on patients with multiple comorbidities whose adverse events and mortality outcomes seem to be similar that those of patients with more advanced breast cancer stages. Similarly, the presence of one or more geriatric syndromes is related to adverse outcomes. The ability to screen and diagnose these issues is of paramount importance for patient stratification. CGA appears to be a critical tool to achieve appropriate and thorough patient evaluation.

\section{Frail breast cancer patients}

Senior breast cancer patients who are frail (as defined by frailty scores) are subject to multiple complications. The presence of geriatric syndromes (falls, incontinence, dementia, depression, nutritional problems) also predisposes senior breast cancer patients to vulnerability and increased mortality outcomes $[45,46]$.

Treatment modalities have not been established for senior frail 
Citation: Ruiz M, Cefalu C, Reske T (2011) Breast Cancer in Senior Patients: Role of Comprehensive Geriatrics Assessment and Comorbidities. J Cancer Sci Ther S2. doi:10.4172/1948-5956.S2-002

breast cancer patients. In the majority of cases, palliative options are offered but decisions take into consideration possible adverse reactions and side effects as opposed to quality of life [47].

Primary endocrine therapy has been suggested for frail breast cancer patients [16]. Single agents are preferred regimens for this population. The specifics of dosing and dose reductions for these patients are still to be determined [45-50]. Generally, management of frail senior breast cancer patients has not been studied in depth. Further it is hypothesized that the frail nature of these patients may cause multiple adverse reactions, complications and adverse outcomes.

\section{Conclusions}

Breast cancer prevalence continues to increase specially in senior patients. It is expected that by the year $2030,60 \%$ of patients will be $>70$ years of age. The Comprehensive Geriatrics Assessment has been advocated as a screening and follow up tool for senior patients. In senior breast cancer patients, CGA may help stratify patients who may develop adverse reactions to treatment strategies. The roles of comorbidities, geriatric syndromes, and frailty status and their impact on survival need to be elucidated.

Frail senior breast cancer patients represent a unique population. Treatment strategies and issues related to quality of life also need to be addressed. The inclusion of frail senior breast cancer patients in future studies is of paramount importance to outline strategies are needed to better care this population.

Finally, the CGA is and will continue to be the gold standard for evaluation of elderly breast cancer patients. Even though shorter versions have been revised issues related to generalizability and validity are common to these tools. The CGA should be included in clinical trials and comparisons between the CGA, and shorter or abbreviated forms are needed for further management of elderly breast cancer patients.

\section{References}

1. Hamaker ME, Schreurs WH, Uppeschoten JM, Smorenburg CH (2009) Breas Cancer in the Elderly: Retrospective Study on Diagnosis and Treatment According to National Guidelines. Breast J 15: 26-33.

2. Livi L, Paiar F, Saieva C, Simontacchi G, Nori J, et al. (2006) Breast Cancer in the Elderly: Treatment of 1500 patients. Breast J 12: 353-359.

3. Hurria A, Togawa K, Mohile S, Owusu C, Klepin H, et al. (2011) Predicting Chemotherapy Toxicity in Older Adults With Cancer: A Prospective Multicenter Study. J Clin Oncol 29: 25.

4. Clough-Gorr K, Stuck A, Thwin S, Silliman R (2010) Older Breast Cancer Survivors: Geriatric Assessment Domains Are Associated With Poor Tolerance of Treatment Adverse Effects and Predict Mortality Over 7 Years of Follow-Up. $\mathrm{J}$ Clin Oncol 28: 380-386.

5. Taira N, Masataka E, Sawaki E, Takahashi M, Shimozuma K, et al. (2010) Comprehensive geriatric assessment in elderly breast cancer patients. Breast Cancer 17: 183-189.

6. Ellis G, Whitehead MA, O'Neill D, Langhorne P, Robinson D (2011) Comprehensive geriatric assessment for older adults admitted to hospital (Review). Cochrane Database Syst Rev CD006211.

7. Schonberg M, Marcantonio E, Ngo L, Li D, Silliman R, et al. (2011) Causes of Death and Relative Survival of Older Women After a Breast Cancer Diagnosis. J Clin Oncol 29: 1570-1577.

8. Foster JA, Salina GD, Mansell D, Williamson JC, Casebeer LL, et al. (2010) How Does Older Age Influence Oncologists' Cancer Management? The Oncologist 15: 584-592.
9. Tucci A, Ferrari SM, Bottelli C, Borlenghi E, Drera M, et al. (2009) A Comprehensive Geriatric Assessment Is More Effective Than Clinical Judgment to Indentify Elderly Diffuse Large Cell Lymphoma Patients Who Benefit from Aggressive Therapy. Cancer 115: 4547-4553.

10. Bernard JR, Vallow LA, DePeri ER, McNeil RB, Feigel DG, et al. (2010) In Newly Diagnosed Breast Cancer, Screening MRI of the Contralateral Breast Detects Mamographically Occult Cancer, Even in Elderly Women: The Mayo Clinic in Florida Experience. Breast J 16: 118-126.

11. Rama VS, Jamel JKA, Mahapatra TK, MCManus PL, Fox JN, et al. (2007) Surgery is Associated with Lower Morbidity and Longer Survival in Elderly Breast Cancer Patients over 80. Breast J 13: 368-373.

12. Molina-Garrido MJ, Guillen-Ponce C (2011) Comparison of two frailty screening tools in older women with early breast cancer. Crit Rev Oncol Hematol 79: 5164.

13. Vacek P, Skelly P, Geller B (2011) Breast cancer risk assessment in women aged 70 and older. Breast Cancer Res Treat 130: 291-299.

14. Dias dos Santos G, Yuka R, Chubaci S (2011) Awareness about breast cance and mammography in elderly women who frequent Daycare Centers in São Paulo (SP, Brazil) Cien Saude Colet 16: 2533-2540.

15. Wang J, Kollias J, Boult M, Babidge W, Zorbas HN, et al. (2010) Patterns of Surgical Treatment for Women with Breast Cancer in Relation to Age. Breast $\mathrm{J}$ 16: $60-65$.

16. Ring A, Reed M, Leonard R, Kunkler I, Wildiers H, et al. (2011) The treatment of early breast cancer in women over the age of 70. Br J Cancer 1-5.

17. Weggelaar I, Aben KK, Warle MC, Strobbe LJ, Van Spronsen DJ (2011) Declined Guideline Adherence in Older Breast Cancer Patients: A PopulationBased Study in the Netherlands. Br J Cancer 17: 239-245.

18. Muss HB, Woolf S, Berry D, Cirrincione C, Weiss R, et al. (2005) Adjuvant Chemotherapy in Older and Younger Women with Lymph Node-Positive Breast Cancer. JAMA 293: 1073-1081.

19. Sautler-Bihi ML, Souchon R, Gerber B (2011) Adjuvant Therapy for Women Over Age 65 With Breast Cancer. Deutsches Artztebiat International 108: 365 371.

20. Freyer G, Campone M, Peron J, Facchini T, Terret C, et al. (2011) Adjuvant docetaxel/cyclophosphamide in breast cancer patients over the age of 70 Results of an observational study. Crit Rev Oncol Hematol [Epub ahead of print].

21. Kimmick G (2011) Adjuvant Chemotherapy for Breast Cancer in Older Women: Emerging Evidence to Aid in Decision Making. Curr Treat Options Oncol 12 286-301.

22. Garg P, Rana F, Gupta R, Buzaianu EM, Guthrie TH (2009) Predictors of Toxicity and Toxicity Profile of Adjuvant Chemotherapy in Elderly Breas Cancer Patients. Breast J 15: 404-408.

23. Balducci L (2009) Treating elderly patients with hormone sensitive breast cancer: What do the data show? Cancer Treat Rev 35: 47-56.

24. Hughes K, Schnaper LA, Berry D, Cirrincione C, McCormick B, et al. (2004) Lumpectomy plus Tamoxifen with or without Irradiation in Women 70 Years of Age or Older with Early Breast Cancer. N Engl J Med 351: 971-977.

25. Spano JP, Falandry C, Chaibi P, Freyer G (2011) Current Targeted Therapies in Breast Cancer: Clinical Applications in the Elderly Woman. Oncologist 16 1144-1153.

26. Hurria A, Gupta S, Zauderer M, Zuckerman EL, Cohen HJ, et al. (2005) Developing a cancer-specific geriatric assessment: a feasibility study. Cancer 104: 1998-2005.

27. Extermann M, Meyer J, MCGinnis M, Croker TT, Corcoran MB, et al. (2004) A comprehensive geriatric intervention detects multiple problems in older breast cancer patients. Crit Rev Oncol Hematol 49: 69-75.

28. Spazzapan S, Crivellari D, Bedar P, Lombardi D, Miolo G, et al. (2011) Therapeutic management of breast cancer in the elderly. Expert Opin Phamarcothe 12: 945-960. 
Citation: Ruiz M, Cefalu C, Reske T (2011) Breast Cancer in Senior Patients: Role of Comprehensive Geriatrics Assessment and Comorbidities. J Cancer Sci Ther S2. doi:10.4172/1948-5956.S2-002

Page 5 of 5

29. Wildiers $H$ (2007) Mastering chemotherapy dose reduction in elderly cancer patients. Eur J Cancer 43: 2235-2241.

30. Cheema FN, Abraham NS, Berger DH, Albo D, Taffet GE, et al. (2011) Nove approaches to perioperative assessment and intervention may improve longterm outcomes after colorectal cancer resection in older adults. Ann Surg 253: 867-874

31. Biesma B, Wymenga AN, Vincent A, Dalesio O, Smit HJ, et al. (2011) Quality of life, geriatric assessment and survival in elderly patients with non-small cell lung cancer treated with carboplatin-gemcitabine-paclitaxel: NVALT-3 a phase III study. Ann Oncol 22: 1520-1527.

32. Aparicio T,Girard L, Bouarioua N, Patry C, Legrain S (2011) A mini geriatric assessment helps treatment decision in elderly patients with digestive cancer: A pilot study. Crit Rev Oncol Hematol 77: 63-69.

33. Overcash JA, Beckstead J, Extermann M, Cobb S (2005) The abbreviated comprehensive geriatric assessment (aCGA): a retrospective analysis. Crit Rev Oncol Hematol 54: 129-136.

34. Hurria A, Lichtman SM, Gardes J, Li D, Limave S, et al. (2007) Identifying vulnerable older adults with cancer: integrating geriatric assessment into oncology practice J Am Geriatr Soc 55: 1604-1608.

35. Molina-Garrido MJ, Guillen-Poince C (2011) Development of a cancer-specific Comprehensive Geriatric Assesesment in a University Hospital in Spain. Crit Rev Oncol Hematol 77: 148-161.

36. Owusu C, Studenski SA (2009) Shared care in geriatric oncology: primary care providers' and medical/oncologist's perspectives. J Am Geriatr Soc 57: S239242.

37. Barthélémy P, Heitz D, Mathelin C, Polesi H, Asmane I, et al. (2011) Adjuvant chemotherapy in elderly patients with early breast cancer. Impact of age and comprehensive geriatric assessment on tumor board proposals. Crit Rev Oncol Hematol 79: 196-204.

38. AlbrandG, Terret C (2008) Early Breast Cancer in the Elderly Assessment and Management Considerations. Drugs Aging 25: 35-45

39. Wedding U, Höffken K (2003) Care of breast cancer in the elderly womanwhat does comprehensive geriatric assessment (CGA) help? Support Care Cancer 11: 769-774.
40. Siegelmann-Danieli N, Khandelwal V, Wood C, Mainalli R, Prichard J, et al (2006) Breast Cancer in the Elderly Women: Outcome as affected by Age Tumor features, Comorbidities, and Treatment Approach. Clinical Breast Cancer 7: 59-66

41. Mohile SG, Xian Y, Dale W, Fisher SG, Rodin M, et al. (2009) Association of a cancer diagnosis with vulnerability and frailty in older Medicare beneficiaries. $J$ Natl Cancer Inst 101: 1206-1215.

42. McCaskill-Stevens W, Abrams JS (2011) Comorbidities in the Aging Breas Cancer Population: Are Current Assessments Leading to Improved Outcomes? J Natl Cancer Inst 103: 1072-1073.

43. Patnaik JL, Byers T, Di Guiseppi C, Denberg TD, Dabelea D (2011) The Influence of Comorbidities on Overall survival Among Older Women Diagnosed with Breast Cancer. J Natl Cancer Inst 103: 1101-1111.

44. Gironés R, Torregrosa D, Díaz-Beveridge R (2010) Comorbidity, disability and geriatric syndromes in elderly breast cancer survivors. Results of a singlecenter experience. Crit Rev Oncol Hematol 73: 236-245.

45. Maas HA, Janssen-Heijnen ML, Olde Rikkert MG, Machteld Wymenga AN (2007) Comprehensive geriatric assessment and its clinical impact in oncology. Eur J Cancer 43: 2161-2169.

46. Reimer T, Gerber B (2010) Quality-of-life considerations in the treatment of early-stage breast cancer in the elderly Drugs Aging 27: 791-800.

47. Aapro M, Monfardini S, Jirillo A, Basso U (2009) Management of primary and advanced breast cancer in older unfit patients (medical treatment). Cancer Treat Rev 35: 503-508.

48. Koroukian SM (2009) Assessment and interpretation of comorbidity burden in older adults with cancer. J Am Geriatr Soc 57: S275-278.

49. Harri E, Hwang T, Urtishak S, Plastaras J, Kinosian B, et al. (2008) The impact of comorbidities on outcomes for elderly women treated with breastconservation treatment for early-stage breast cancer. Int J Radiat Oncol Bio Phys 70: 1453-1459

50. Yancik R, Wesley M, Ries L, Havlik R, Edwards B, et al. (2001) Effect of Age and Comorbidity in Postmenopausal Breast Cancer Patients Aged 55 Years and Older. JAMA 285: 885-892. 